\title{
F-18 FDG PET-CT in a Case of Unsuspected Bilateral Adrenal Histoplasmosis
}

\author{
Thanuja $M^{\mathrm{a}}$, Fazarina $\mathrm{M}^{\mathrm{b}}$, Ding $\mathrm{CH}^{\mathrm{c}}$ \\ ${ }^{a}$ Department of Molecular Imaging and Nuclear Medicine, Universiti Kebangsaan Malaysia Medical Centre \\ ${ }^{b}$ Department of Laboratory Diagnostic Services, Universiti Kebangsaan Malaysia Medical Centre \\ ${ }^{c}$ Department of Medical Microbiology and Immunology, Faculty of Medicine, Universiti Kebangsaan Malaysia
}

\begin{abstract}
Histoplasmosis is a common fungal infection in endemic countries including Malaysia. Although it is commonly diagnosed in immunosuppressed individuals, there is a preponderance of immunocompetent individuals among individuals with disseminated disease involving adrenal gland. There is a diagnostic dilemma in most patients as the clinical presentation, biochemical parameters and imaging features are frequently non-specific. On 18F-FDG PETCT, histoplasmosis shows high FDG avidity. However, due to overlapping features with other disease conditions, tissue biopsy remains as the confirmatory test. In this report, we present a case of disseminated histoplasmosis with high FDG uptake in the adrenal glands and lymph nodes which mimic a metastatic cancer.
\end{abstract}

KEYWORDS: F-18 FDG PET-CT, adrenal gland, histoplasmosis

\section{INTRODUCTION}

A Fluorine-18 Fluorodeoxyglucose Positron Emission Tomography-Computed Tomography (F-18-FDG PETCT) has an important role in the management of various benign and malignant conditions of the adrenals. In patients who present with bilateral adrenal masses, FDG PET-CT is performed for further characterization of these masses and usually to exclude malignant disease. However, there is a possibilities of false-positive interpretations as there is an overlapping features between benign and malignant lesions, both morphologically and metabolically. The benign adrenal conditions that cause bilateral symmetrical FDG uptake are adrenal hyperplasia, tuberculosis and fungal infections. In this report, we present a case of bilateral adrenal histoplasmosis which shown an intense FDG uptake and was initially misinterpreted as malignant

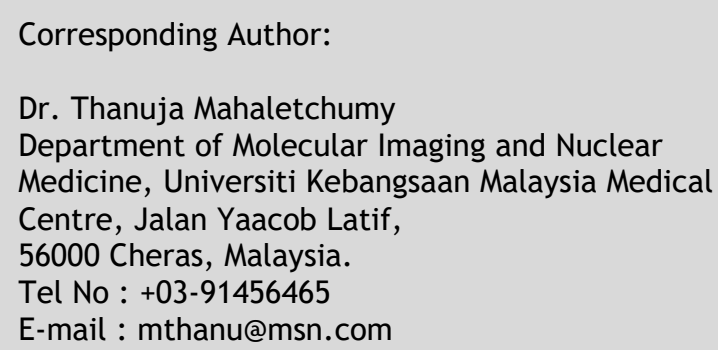

masses. Our case highlights a histoplasmosis case which cause false-positive findings on FDG PET/CT performed for malignancy in an immunocompetent patient.

\section{CASE REPORT}

A 78-year-old gentleman with underlying diabetes mellitus, hypertension and dyslipidaemia presented with generalized weakness, hoarseness of voice, polyuria, dysphagia, loss of appetite and weight loss of $11 \mathrm{~kg}$ in 2 months. An initial investigations shown raised serum calcium. Otherwise his full blood count, tumour markers, inflammatory markers, septic work-up, Mantoux test and chest $\mathrm{x}$-ray were all normal. His contrast enhanced computed tomography (CT) of thorax and abdomen shown bilateral enlargement of adrenal glands with heterogenous enhancement and central hypodensity. There was also saccular aneurysm of the arch of aortic arch. Laryngoscopy revealed right vocal cord palsy which was attributed to the aortic aneurysm. In view of negative biochemical parameters for infection and inflammation, adrenal metastases from unknown primary was suspected. Subsequently, A 
Fluorine-18 Fluorodeoxyglucose Positron Emission Tomography-Computed Tomography (F-18-FDG PET -CT) was performed for further characterization of the adrenal masses and to identify possible occult primary malignancy. Fifty minutes after intravenous injection of 191MBq F-18-FDG, a whole body scan PET/CT scan was performed. The scan revealed high Fluorodeoxyglucose (FDG) uptake in both of the adrenal masses with maximum standardized uptake value (SUVmax) of 15.6 (Fig. 1a). There was also increased FDG uptake in a few submental and submandibular nodes with SUVmax ranging between 7.3 to 12.8 (Fig. 1b, 1c). There was hepatosplenomegaly which showed physiological FDG uptake. There was no abnormal uptake in other sites to suggest a primary lesion. The differential diagnoses at this time included tuberculosis (TB), lymphoma and metastasis from unknown primary malignancy. A CT guided biopsy of the right adrenal gland was performed. The microscopic images from the adrenal biopsy showed abundant intracellular small ovoid yeast-like organisms measuring 2-4 micrometers periodic acid-Schiff (PAS) and Grocott methenamine-silver (GMS) stain positive with occasional narrow based budding, clear halo and presence of clustering within macrophages (Fig. 2a, 2b). Microscopic features were suggestive of histoplasmosis. Based on the constellation of findings, the diagnosis of disseminated histoplasmosis was made. Patient subsequently developed adrenal insufficiency and was treated with intravenous hydrocortisone. However, patient's family requested for a discharge prior to initiation of anti-fungal treatment. Two months later, he succumbed to his illness.

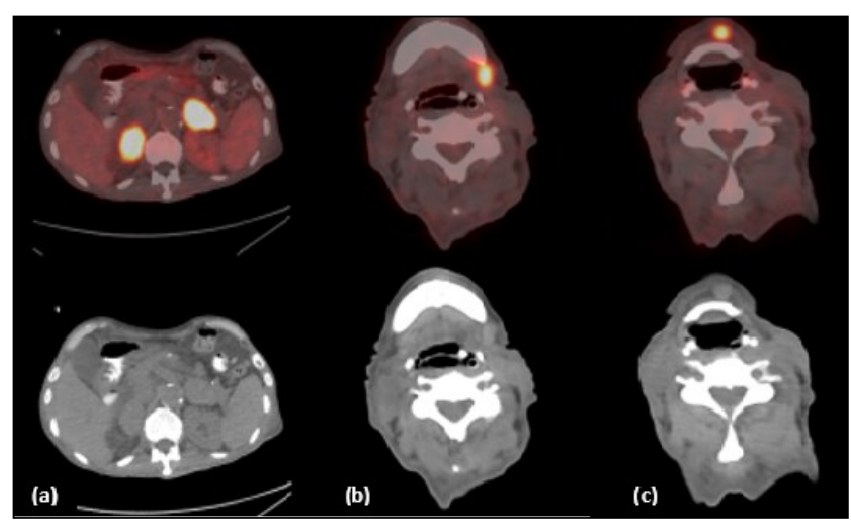

Figure 1: Axial FDG PET-CT images showing high FDG uptake in (a) bilateral adrenal glands, (b) left submandibular node and (c) submental node. 
Other systems that are frequently involved in histoplasmosis are the reticuloendothelial system manifested as hepatosplenomegaly and bone marrow involvement resulted in pancytopenia. In our patient, there was evidence of hepatosplenomegaly which is likely due to involvement of reticuloendothelial system in histoplasmosis. Presence of hypercalcemia is linked to an increased in 1, 25 dihydroxyvitamin $\mathrm{D}$ production from the fungal granulomas was seen in this case as well as other studies.6,7 The FDG PET-CT scan which was performed in our patient also showed increase in metabolic activity in the submental and submandibular nodes. Even though no cytological assessment of the lymph nodes was done, we think that an increase in metabolic activity was attributed to histoplasmosis involvement. We found a case report which had similar findings with involvement of bilateral adrenal glands and submandibular nodes, in which was confirmed cytologically. ${ }^{8}$

F-18 FDG PET-CT has revolutionized the diagnosis, staging, monitoring response to treatment and followup of patients with suspected recurrence in myriad of malignancies. Owing to its non-specific tracer properties, FDG accumulation is seen not only in malignant cells but also in infectious tissues due to presence of migratory inflammatory cells, microorganisms, and granulation tissue.? This finding enables the identification of the sites of infection, assessment of the extension of the disease involvement and monitor response to treatment. However, the use of FDG PET-CT in the diagnosis and management of infections is still limited due to lack of adequate evidence as well as supporting literature. The limitation is also due to overlap of imaging features both morphologically and metabolically with primary and metastatic malignant conditions. The typical FDG PET -CT findings of adrenal histoplasmosis are intensely FDG avid bilateral adrenal enlargement. ${ }^{9}$ On a contrastenhanced FDG PET-CT, the adrenals will show low density areas of central necrosis/hemorrhage and peripheral enhancing rim on the CT component. The presence of central hypodensity is a common imaging feature for a few other disease conditions including metastasis, tuberculosis and other fungal infections such coccidiodomycosis and blastomycosis. However, the presence of central hypodensity with peripheral enhancement typically occurs only in tuberculosis and histoplasmosis. ${ }^{10}$ In our case, there was suspicion of malignancy initially due to the presence of intensely FDG avid bilateral enlarged adrenal glands with heterogenous enhancement and central hypodensity on CT.

Due to an overlap in clinical presentation and imaging features on FDG PET-CT in a case of bilateral enlarged adrenal glands between adrenal histoplamosis and other differential diagnosis such as metastasis, lymphoma, tuberculosis or other fungal infections, a high index of suspicion is required especially in an immunocompetent host. At times, since histoplasmosis is not considered to be part of the differential diagnosis, it may lead to unnecessary delay in initiation of treatment leading to adrenal insufficiency. Therefore, histopathological assessment has to be performed for a definite diagnosis.

\section{CONCLUSION}

As both benign and malignant adrenal lesions can show increased FDG uptake on FDG PET/CT, it is prudent that careful correlation with patient's history, biochemical parameters and histopathological assessment is done. Based on our case report and few other cases that have been reported in literature, adrenal histoplasmosis is a differential diagnosis that has to be considered in an immunocompetent patient who presents with bilateral/unilateral adrenal masses and especially in the absence of definite primary lesion on FDG PET-CT

\section{REFERENCES}

1. Jacob Baker, Findra Setianingrum, Retno Wahyuningsih \& David W. Denning (2019) Mapping histoplasmosis in South East Asia implications for diagnosis in AIDS, Emerging Microbes \& Infections, 8:1, 1139-1145,

2. Shahar, MA, A. Wahab@A. Rahman, N, AbAziz, A, Ahmad Marzuki, O, Mohd Rusly, NA \& Kamaruddin, NA 2015, 'Adrenal insufficiency resulting from Histoplasmosis infiltration in an immunocompetent patient', Brunei International Medical Journal, vol. 11, no. 5, pp. 261-264.

3. Benevides CF, Duraes RO, Aquino B, Schiavon 
Lde L, Narciso-Schiavon JL, Buzzoleti Fda C.

Bilateral adrenal histoplasmosis in an

immunocompetent man. Rev Soc Bras Med

Trop. 2007; 40: 230-3.

4. Bhansali A, Das S, Dutta P, et al. Adrenal

histoplasmosis:unusual presentations. J Assoc

Physicians India. 2012; 60:54-8.

5. Gajendra S, Sharma R, Goel S, et al. Adrenal

Histoplasmosis in Immunocompetent Patients

Presenting as Adrenal Insufficiency. Turk

Patoloji Dergisi. 2016;32(2):105-111.

6. Liu JW, Huang TC, Lu YC, et al. Acute

disseminated histoplasmosis complicated with

hypercalcaemia. J Infect. 1999;39(1):88-90.

7. Khasawneh FA, Ahmed S, Halloush

RA. Progressive disseminated histoplasmosis presenting with cachexia and hypercalcemia. Int J Gen Med 2013; 6: 79-83

8. Salim, Nelda \& Tanzil, Iswadi \& Permata, Mega \& Hudari, Harun \& Perdana, Rizky. (2019).

Histoplasmosis of bilateral adrenal masses and submandibular lymphadenopathy: other diagnosis besides malignancy and tuberculosis. Journal of Physics: Conference Series. 1246. 012052. 10.1088/1742-6596/1246/1/012052.

9. Sharma P, Mukherjee A, Karunanithi S, Bal C, Kumar R. Potential role of 18F-FDG PET/CT in patients with fungal infections. AJR Am J Roentgenol. 2014;203:180-9.

10. Kawashima A, Sandler CM, Fishman EK et al. Spectrum of CT findings in nonmalignant disease of the adrenal gland. Radiographics 1998; 18: 393- 412. 